\title{
Influência das características coercitiva e habilitante do orçamento no empoderamento e na criatividade
}

\author{
Thiago Bruno de Jesus Silva \\ https://orcid.org/0000-0002-1128-6601 | E-mail:thiagobruno.silva@yahoo.com.br \\ Cristian Baú Dal Magro \\ https://orcid.org/0000-0002-7609-5806 | E-mail:crisbau@unochapeco.edu.br \\ Luciana Goncalves de Souza \\ https://orcid.org/0000-0001-6670-9539 ｜ E-mail: lucianasouza.contadora@gmail.com \\ Januario Jose Monteiro \\ https://orcid.org/0000-0002-7000-4256 | E-mail:januariomonteiromonteiro@gmail.com
}

\section{Resumo}

Objetivo: Analisar a influência das características coercitiva e habilitante do orçamento no empoderamento e na criatividade dos gestores.

Método: Efetuou-se uma survey com 100 respostas válidas de gestores pertencentes à Associação das Empresas Brasileiras de Tecnologia da Informação (Assespro). Os dados foram analisados a partir da modelagem de equações estruturais, com uso do PartialLeastSquares (PLS).

Resultados: Os resultados sugerem que as características coercitivas do orçamento aumentam a criatividade dos gestores de empresas tecnológicas. De outro lado, percebeu-se que as influências das características habilitantes do orçamento na criatividade foram significativas e maiores do que as das características coercitivas. Em relação ao teste de mediação, verificou-se que o empoderamento não mediou a relação entre as características coercitivas do orçamento (planejamento) e a criatividade, mas o empoderamento mediou parcialmente a relação entre as características habilitantes do orçamento (diálogo) e a criatividade. Contribuições: Esta investigação contribui com o campo teórico ao demonstrar que o orçamento empresarial oferece possibilidade de ser percebido de duas formas distintas, o que pode contribuir para o sentimento de empoderamento e a criatividade dos funcionários, facilitando o consumo de recursos de maneira sustentável e inovadora pelas iniciativas criativas dos funcionários.

Palavras-chave: Controle Coercitivo; Controle Habilitante; Utilidade do Orçamento; Empoderamento; Criatividade. 


\section{Introdução}

O orçamento é um instrumento utilizado para cumprir os objetivos de planejamento (planejamento, coordenação, alocação de recursos e determinação dos volumes operacionais) e diálogo (comunicação, criação de consciência, motivação) (Ekholm \& Wallin, 2011). A teoria da formalização burocrática pode estar em consonância com a utilidade do orçamento (Adler \& Borys, 1996). Nesse sentido, o orçamento útil ao planejamento apresenta forma coercitiva (coercive), por oferecer processos rígidos e com pouca utilidade interativa (Radke \& Widener, 2016).

Isso denota um controle orçamentário adequado ao cumprimento de padrões previamente especificados (Ahrens \& Chapman, 2004). O estudo argumenta que o uso do orçamento cria um ambiente caracterizado pela autogestão e a liberdade dentro dos limites estipulados, o que promove o empoderamento (Simons, 1994). Mundy (2010) comenta que o uso do orçamento pelo atributo do diálogo fornece clareza e entendimento do ambiente de trabalho.

Estudos recentes forneceram evidências empíricas de que quando controles gerenciais diferentes têm pesos diferentes no sistema, suas combinações apoiam a criatividade (Henri 2006; Widener 2007; Mundy 2010; Bedford, 2015; Kruis et al., 2016; Curtis \& Sweeney, 2017; Janka \& Guenther 2018; MullerStewens et al., 2020). O orçamento pautado no diálogo pode ser vinculado ao modelo habilitante (enabling) por oferecer responsabilidade e autonomia aos funcionários (Radke \&Widener, 2016).

Desse modo, as utilidades do orçamento com características habilitantes permitem que haja o diálogo para possíveis decisões e estimula a interação entre os entes da hierarquia em uma organização (Adler \& Borys, 1996). A criatividade dos colaboradores tem sido crucial para o sucesso das organizações e, portanto, a iniciativa de implementação de controles gerenciais, por vezes, tem sido um dilema para este processo (Amabile, Conti, Coon, Lazenby e Herron, 1996).

O dilema enfrentado pelas empresas se vincula ao fato de que a natureza específica da produção criativa requer o uso substancial do controle formal, mas esse controle pode prejudicar a criatividade (Grabner \& Speckbacher, 2016). Contudo, o comportamento disfuncional requer controle formal para coordenar e regular efetivamente o comportamento dos funcionários (Adler \& Chen, 2011).

Nesse contexto, o controle orçamentário permite que as metas fiquem alinhadas ao comportamento dos funcionários para que haja maior eficiência (Speklé, Elten \& Widener, 2017). A literatura comportamental indica que a liberdade no trabalho, que pode ser facilitada pelos controles gerenciais, permite que os indivíduos sejam criativos e, por outro lado, controles rígidos podem se tornar um impedimento ao pensamento criativo (Amabile et al., 1996).

A literatura sugere que o comportamento dos gestores é afetado pela atuação dos controles formais na comunicação das restrições, limites e do poder no desenvolvimento das tarefas (Koestner, Ryan, Bernieri \& Holt, 1984). Além disso, o controle orçamentário pode oferecer a sensação de empoderamento ao funcionário, por indicar que as suas escolhas e expectativas podem ser orientadas pelo seu esforço. Os comportamentos autorregulados podem ser apoiados pelo empoderamento, conforme exposto na teoria da Autodeterminação (Sierens, Vansteenkiste, Goossens, Soenens \& Dochy 2009).

É importante entender os efeitos do orçamento com característica coercitiva e habilitante na criatividade e no empoderamento dos gestores. Evidências recentes indicam que o Sistema de Controle Gerencial (SCG) pode favorecer o empoderamento e a criatividade (Adler \& Chen, 2011; Marginson, Aulay, Roush \& Zijl, 2014; Grabner e Speckbacher, 2016; Cools, Stouthuysen \& Van Abbeele, 2017; Speklé et al., 2017), contudo, como se comporta o controle orçamentário neste processo, tendo em vista que é um componente do Sistema de Controle Gerencial. 
As informações do SCG podem ser usadas na forma coercitiva para garantir que a organização está no caminho certo para alcançar os objetivos pretendidos, mas também podem ser usadas de forma habilitante para garantir que as informações e preocupações da alta gerência são compartilhadas verticalmente (Muller-Stewens, Widener, Moller \& Steinmann, 2020). Nesse sentido, este estudo teve o seguinte problema de pesquisa: Qual a influência das características coercitiva e habilitante do orçamento no empoderamento e na criatividade dos gestores? O estudo tem o objetivo de analisar a influência das características coercitiva e habilitante do orçamento no empoderamento e na criatividade dos gestores.

Esta investigação contribui com o campo teórico ao demonstrar que o orçamento empresarial oferece possibilidade de ser percebido de duas formas distintas, o que pode contribuir para o sentimento de empoderamento e a criatividade dos funcionários, facilitando o consumo de recursos de maneira sustentável e inovadora pelas iniciativas criativas dos funcionários. Os resultados do estudo sugerem que a utilização do orçamento, por si só, é um instrumento que oferece maior criatividade organizacional. Nesse aspecto, têm-se práticas contábeis que conciliam características mecanicistas e orgânicas (Ahrens \& Borys, 1996).

No aspecto prático, o estudo contribui por propiciar informações sobre o processo de gestão, o qual oferece instrumentos adequados à continuidade dos negócios. As principais evidências sugerem que o orçamento de forma habilitante tem maior facilidade de promover a criatividade organizacional, quando comparado com o coercitivo. Essas utilidades permitem diálogo para possíveis decisões, estimula a interação entre os entes da hierarquia (Ahrens \& Chapman, 2004) e desenvolve autonomia e aprendizagem (Radker \& Widener, 2016).

\section{Fundamentação Teórica e Desenvolvimento das Hipóteses}

\subsection{Utilidades do Orçamento e Duas tipologias de Formalização}

O orçamento empresarial exerce diversas utilidades que podem ser caracterizadas como controle cibernético, para sinalizar metas, analisar desvios e fazer correções (Kruis, Widener \& Speklé, 2016), bem como para influenciar comportamentos, motivar, gerar debate e disseminar conhecimento para toda a organização (Arnold \& Gillenkirch, 2015).

Ekholm e Wallin (2011) argumentam que o orçamento tem duas utilidades nas organizações, sendo: planejamento (planejamento, coordenação, alocação de recursos e determinação dos volumes operacionais) e diálogo (comunicação, criação de consciência, motivação). As utilidades destacadas fornecem possibilidade informativa, o que propõem a reconciliação entre aspectos positivos e negativos da burocracia de acordo com as duas tipologias de formalização, que são: coercitiva e habilitante. As utilidades do orçamento são analisadas segundo o framework de Adler e Borys (1996), os quais estudaram as características da formalização, o processo de design e a implementação do sistema.

Complementarmente, o desenho e o uso dos controles gerenciais afetam o comportamento organizacional (Hall, 2008). Os sistemas habilitantes podem facilitar as responsabilidades (Adler \& Borys, 1996; Burney, Radtke \& Widener, 2017), diferente do que ocorre com os dispositivos de controle para uso da alta administração (Free, 2007; Chapman \&Kihn, 2009). O controle gerencial pela formalização habilitante tem poder sobre o comportamento dos indivíduos (Hempel, Zhang \& Han, 2012), gerando um sentimento de liberdade por parte dos funcionários em relação às regras e aos sistemas existentes (Wouters \& Wilderom, 2008). Além disso, proporcionam maior interação entre os seus usuários com o próprio sistema e também entre gestores de diferentes níveis hierárquicos, estimulando a resolução de problemas por oferecer feedback e revelar melhorias (Adler \& Borys, 1996; Wouters \& Wilderom, 2008; Hartmann \& Maas, 2011). 
Adler e Borys (1996) preveem que a formalização habilitante consiste em procedimentos que auxiliam os funcionários a lidar com as contingências no seu local de trabalho para que possam completar suas tarefas e fornece memória organizacional que capta as lições aprendidas com a experiência. A tipologia habilitante permite que, mesmo com as regras, os funcionários se sintam motivados (Wouters \& Wilderom, 2008).

Dessa forma, as regras buscam facilitar a estrutura e refinar a condução do trabalho dos funcionários sem comprometimento hierárquico (Adler \& Borys, 1996). Van der Hauwaert e Bruggeman (2015) sugerem que os controles gerenciais habilitantes auxiliam na criação de um ambiente organizacional capaz de incrementar a motivação, a competência e os relacionamentos.

Os mecanismos de controle, como o orçamento empresarial, se categorizam em uma espécie de continuum de habilitante versus coercitivo (Sánchez, Velez \& Ramón-Jerónimo, 2012). O processo orçamentário se caracteriza como coercitivo no momento em que restringe a liberdade na ação gerencial, utilizado para apresentar forma de como os funcionários e gerentes deverão se comportar.

O orçamento pode ser considerado como habilitante quando permite diálogo para possíveis decisões e estimula a interação entre os entes da hierarquia (Ahrens \& Chapman, 2004). A tipologia de formalização coercitiva busca a condução dos funcionários à conformidade (Wouters \& Wilderom, 2008). Ou seja, são regras com o intuito de conduzir, de forma forçada, o cumprimento de padrões que foram especificados (Ahrens \& Chapman, 2004).

Dessa forma, entende-se que as informações inerentes ao orçamento podem servir para diferentes utilidades, o que possibilita o cumprimento de padrões pré-especificados no planejamento (coercitivo) e oferece diálogo (habilitante). Em síntese, o uso coercitivo do orçamento pode se restringir no controle comportamental dos funcionários e o seu uso habilitante desenvolve a autonomia e a aprendizagem (Radke \& Widener, 2016).

\subsection{Empoderamento e Criatividade}

A teoria da autodeterminação explica como as percepções dos indivíduos sobre estímulos à ação e contextos de decisão influenciam o comportamento intencional e, em particular, seu envolvimento intrínseco e comprometimento com as ações e os esforços (Ryan \& Deci, 2000). O envolvimento positivo com uma tarefa depende de um senso de autodeterminação, que por sua vez depende da satisfação de três necessidades humanas (autonomia, competência e relacionamento), como explicam Deci e Ryan (1987).

$\mathrm{Na}$ autonomia existe a crença de que as ações são próprias e não dependentes da perseverança do indivíduo. Na competência, as pessoas acreditam na sua própria capacidade para executar as tarefas e no seu grau de controle sobre os resultados de suas ações. Já o relacionamento denota um ambiente social que traz uma sensação de segurança e apoio (Ryan \& Deci, 2000).

No local de trabalho, o conceito de autodeterminação se relaciona com a noção de empoderamento, determinando até que ponto os funcionários acreditam que podem executar suas tarefas com autonomia (Speklé et al., 2017). Esta teoria associa diversos aspectos comportamentais à motivação da autodeterminada, incluindo o desenvolvimento social e o bem-estar das pessoas (Ryan \& Deci, 2000).

Nesse sentido, o empowerment psicológico é compreendido pela motivação intrínseca de um indivíduo em relação ao seu ambiente de trabalho, manifestado pelas cognições de significado, competência, autodeterminação e impacto (Spreitzer, 1995). Pesquisas como as de Hall (2008), Mahama e Cheng (2013), Appuhami (2017), Souza, Anzilago e Beuren (2017) ofereceram inferências concludentes sobre os efeitos dos controles gerenciais nas dimensões do empowerment psicológico. Por outro lado, Beuren, Santos e Bernd (2020) sugerem que a percepção habilitante dos controles gerenciais está associada com o empowerment psicológico. Este achado indica que as características dos controles gerenciais afetam o trabalho dos gestores. 
Nesse sentido, a forma de uso do orçamento pelas empresas pode mudar o senso de desenvolvimento social e o bem-estar dos colaboradores. A forma de uso do controle gerencial pode motivar a autodeterminação para diferentes comportamentos, inclusive na produção de ideias criativas (Amabile et al., 1996). A criatividade pode ser induzida pelo empoderamento e pelos sentimentos de propriedade e controle sobre o próprio trabalho (Amabile et al., 1996). Esses autores ainda sugerem que o empoderamento encoraja os funcionários ao uso de formas alternativas para a execução do trabalho.

Annarelli e Nonino (2016) comprovaram que o controle gerencial habilitante afeta a resiliência organizacional, entendida pela capacidade da organização se preparar, responder e se recuperar de eventos inesperados. Nesse sentido, a resiliência organizacional ocorre em função da atitude e do engajamento dos indivíduos que atuam dentro da empresa e, portanto, a criatividade desses mesmos indivíduos pode ser reforçada pelo controle gerencial habilitante.

Limnios et al. (2014) destacam que a resiliência dependerá das características do sistema organizacional e da sua capacidade de interagir com o ambiente de forma ofensiva (adaptativa) ou defensiva (reativa). Nesse sentido, a resiliência pode ser promovida pela criatividade dos indivíduos a partir das características do sistema organizacional. Presume-se que o controle gerencial com característica flexível favorece a realização de mudanças e auxilia os indivíduos a terem maior criatividade para enfrentar os desafios vivenciados diariamente pelas organizações.

\subsection{Desenvolvimento das Hipóteses de Pesquisa}

O uso do orçamento cria um ambiente que é caracterizado pelo autogerenciamento e pela liberdade dentro dos limites estipulados, o que promove o empoderamento (Simons, 1994). Annarelli e Nanino (2006), Mundy (2010), Limnios et al. (2014) sustentam este argumento ao indicarem que os controles gerenciais são importantes para direcionar e capacitar gestores e funcionários de forma simultânea, o que pode facilitar a criatividade e ao mesmo tempo restringir comportamentos impróprios.

A utilidade do orçamento agrupada no planejamento tem sido caracterizada pelo controle cibernético (Bedford, 2015). Assim, pode ser ligada a perspectiva cibernética do controle, o que é imprescindível para planejar de forma operacional e avaliar o desempenho (Arnold \& Artz, 2019). Ou seja, são conduções com a intenção de direcionar o cumprimento de padrões que foram definidos (Ahrens \& Chapman, 2004).

Nesse cenário, Wouters e Roiimans (2011) indicam que o estabelecimento de metas pode encorajar a resolução de problemas e a experimentação. Grabner e Speckbacher (2016) sugerem que objetivos predefinidos são utilizados na avaliação de desempenho em ambientes criativos. Para tanto, trabalhar em direção à meta oferece autonomia para promover a criatividade da equipe, desenvolvendo soluções inovadoras para garantir a perpetuidade organizacional (Amabile, 1997; Cools et al., 2017).

Propõe-se que os aspectos indicados sejam consequência da criação de um ambiente que forneça suporte à autonomia para promover motivação intrínseca e comportamentos auto-reguladores, em oposição a um ambiente caracterizado como "controlador" (Simons, 1995). Diante das exposições, os funcionários podem ter a oportunidade de fazer escolhas sobre suas ações dentro de limites (Amabile et al., 1996).

Cools et al. (2017) concluem que o orçamento voltando ao planejamento elucida as metas, os objetivos e as restrições que levam à estruturação do problema de decisão para que o pensamento criativo seja preciso, porque as soluções não se ajustam a todos os parâmetros de decisão (Speklé et al., 2017). Para os autores, também podem servir para fornecer orientação e melhorar a compreensão das pessoas sobre a relação entre ação e resultado, o que guia ainda mais o comportamento criativo. Deste modo, propõese a seguinte hipótese:

- H1 - As utilidades do orçamento agrupadas no planejamento influenciam a criatividade. 
Pesquisas em psicologia social e comportamento organizacional se atentaram a vários vetores de criatividade (Abbey \& Dickson, 1983; Amabile et al., 1996; Hirst, van Knippenberg \& Zhou, 2009). Estes estudos concluíram que os sentimentos de autonomia são importantes, mas o próprio ambiente de trabalho também desempenha um papel significativo (Amabile \& Pillemer, 2012).

As utilidades do orçamento agrupadas no diálogo são caracterizadas como controle orgânico (Bedford, 2015). O orçamento, como mecanismo de controle habilitante, permite o diálogo para possíveis decisões, estimula a interação entre os entes da hierarquia (Ahrens \& Chapman, 2004) e, apesar das regras, faz com que os funcionários se sintam motivados (Wouters \& Wilderom, 2008).

Nesse quesito, dar instruções explícitas, reconhecer o pensamento criativo, incentivar um discurso que apoia a criatividade e a geração de ideias, são todos aspectos que oferecem incentivos (Amabile et al., 1996). Essas utilidades do orçamento permitem a troca de informações em um ambiente no qual os indivíduos são incentivados a desafiar o status quo e a participar de debates e diálogos com a finalidade de descobrir soluções criativas para os desafios diários.

As organizações que buscam facilitar a criatividade devem criar um processo de troca de informações que incentive e apoie essa criatividade. As evidências empíricas encontradas por Simons (1994) e Gong, Cheung, Wang e Huang (2012) sugerem que a troca de informações é necessária para que as equipes sejam mais criativas. Nesse sentido, é proposta a seguinte hipótese:

\section{- H2 - As utilidades do orçamento agrupadas no diálogo exercem maior influência na criatividade do que as utilidades agrupadas no planejamento.}

Sanchez, Velez e Ramón-Jerónimo (2012) explicaram sobre a categorização em uma espécie de continuum que vai de habilitante versus coercitivo. O orçamento, como mecanismo de controle, exerce distintas utilidades, como a imposição de limites para a liberdade na ação gerencial e também promove o diálogo, a interação e estimula a motivação (Radke \& Widener, 2016; Ahrens \& Chapman, 2004). Este estudo propõe que os usos coercitivo e habilitante do orçamento forneçam suporte à autonomia.

As utilidades do orçamento agrupadas no planejamento comunicam a meta em medidas críticas de desempenho que servem para orientar o comportamento e fornecer feedback para facilitar o aprendizado (Simons, 1995). Assim, o oferecimento de oportunidades para os funcionários acreditarem que podem fazer escolhas em relação às suas ações dentro de limites definidos (como apresentado no empoderamento) e o incentivo organizacional e de supervisão foram considerados um estimulante imprescindível para a criatividade nos ambientes organizacionais (Amabile et al., 1996).

Além disso, o empoderamento representa um tipo de motivação autônoma por apoiar a autonomia dos indivíduos (Gagne \& Deci, 2005). Para empoderar é preciso facilitar e ampliar o acesso informacional em diferentes níveis (Spreitzer, 1995). Sendo assim, o compartilhamento de informações fortalece o senso de competência e propósito do indivíduo, tornando-o um elemento valioso para a organização (Spreitzer, 1995).

Algumas pesquisas na área contábil (Hall, 2008; Mahama \& Cheng, 2013; Mouang, 2015; Appuhami, 2017; Souza et al., 2017; Souza \& Beuren, 2018) sugerem que o desenho e o uso dos controles gerenciais interferem no empoderamento dos indivíduos. Hempel et al. (2012) aduzem que a formalização organizacional pode promover o empoderamento, por ser capaz de direcionar a organização, independentemente de ser ou não centralizada.

Sendo assim, os controles gerenciais habilitantes podem minimizar as disparidades de poder na organização, pela ampliação do compartilhamento de conhecimento, habilidades e recompensas entre os níveis organizacionais (Adler \& Borys, 1996). A ampliação dos limites de tomada de decisão dos funcionários reflete no empoderamento, na autoestima e na determinação nas atividades organizacionais (Kantur \& Iseri-Say, 2012). Os indivíduos se sentirão com maior empoderamento por se perceberem como parte significativa da organização, o que implica a formalização mínima de tarefas pela criação de metas e responsabilização (Spreitzer, 1996). Nesse sentido, propõe-se a seguinte hipótese: 


\section{- H3 - O empowerment medeia a relação entre as utilidadesdo orçamento agrupadas no planejamento e a criatividade.}

Estudos recentes forneceram evidências de que quando os controles têm pesos diferentes no sistema de controle gerencial suas combinações suportam a criatividade (Kruis et al., 2016; Bedford, 2015). O orçamento, nas utilidades agrupadas no diálogo, comunica as preocupações dos principais gerentes em todas as unidades organizacionais. Estes funcionários ficam cientes das oportunidades e ameaças potenciais ao atingimento das metas (Speklé, Elten \& Widener, 2017).

As utilidades do orçamento agrupadas no diálogo podem estimular os indivíduos a pensarem em soluções incomuns e abordagens fora do padrão (Burroughs, Dahl, Moreau, Chattopadhyay \& Gorn 2011; Cools et al., 2017). Marginson et al., (2014) sugeriram que o uso do orçamento com utilidades de forma interativa pode ampliar a sensação de empoderamento. Hall (2008) explica que o uso de um conjunto diversificado de medidas de desempenho nas diversas unidades de negócio faz com que os gestores experimentem maior liberdade, autonomia e oportunidade na realização das tarefas.

As pesquisas anteriores apoiam o argumento de que os funcionários se tornam mais criativos quando se sentem empoderados e percebem que têm liberdade para realizar as tarefas (Alge, Ballinger, Tangirala e Oakley, 2006). A consequência disso é que, se a organização está interessada em estimular a criatividade, o ambiente de trabalho deve ser baseado em percepções de empoderamento. Assim, dar instruções explícitas, reconhecer o pensamento criativo, incentivar o discurso que apoia a criatividade e a geração de ideias são todos aspectos do incentivo organizacional de estímulo à criatividade (Amabile et al., 1996; Alge et al., 2006).

Além de oferecer oportunidades para os funcionários acreditarem que podem fazer escolhas sobre suas ações dentro de limites definidos (conforme apresentado no empoderamento), incentivos organizacionais e de supervisão foram considerados um estimulante indispensável para a criatividade organizacional (Amabile et al., 1996).

Consequentemente, o sistema de controle gerencial é projetado e apresentado de forma a ser internalizado e assimilado, o que apoia e aprimora a autodeterminação e, portanto, a percepção de empoderamento. Dessa forma, requer a estrutura de controle gerencial para apoiar a autonomia (Deci \& Ryan, 1987) e fornecer uma estrutura adequada para os problemas de decisão que os indivíduos enfrentam (Sierens et al., 2009). Em um ambiente de trabalho facilitador (interativo), é fácil promover o comportamento criativo, emergindo também a compreensão de que a liberdade pode afetar o nível de empoderamento dos gerentes. Hall (2008) indica que o uso de um conjunto diversificado de medidas de desempenho nas diversas unidades de negócios faz com que os gerentes experimentem maior liberdade e autonomia na execução das tarefas.

Marginson et al. (2014) encontraram um resultado positivo entre o uso interativo de medidas de desempenho não financeiro e a autodeterminação, sugerindo que o uso interativo do orçamento pode melhorar o senso de empoderamento, o qual está associado à motivação intrínseca e autodeterminada.

Usar o orçamento de maneira interativa cria o ambiente de trabalho em que os funcionários precisam ser criativos. $\mathrm{O}$ uso diagnóstico, as metas, os objetivos e limites de desempenho não precisam ser vistos como restrições negativas, mas podem, na presença de controle interativo de suporte, ser percebidos como desafios que apenas geram problemas instigantes, estimulando os indivíduos a pensar em coisas incomuns (Burroughs, Dahl, Moreau, Chattopadhyay \& Gorn 2011; Cools et al., 2017). Ciente desse contexto, o estudo propõe a seguinte hipótese:

- H4 - O empowerment medeia a relação entre as utilidades do orçamento agrupadas no diálogo e a criatividade. 


\section{Procedimentos Metodológicos}

Uma pesquisa de levantamento foi realizada com gestores pertencentes à Assespro. Os dados das organizações são disponibilizados no site de cada Assespro regional (São Paulo, Bahia, Sergipe, Pernambuco, Paraíba, Rio Grande do Sul, Brasília). O instrumento de pesquisa foi aplicado por meio telefônico, no decorrer dos meses de dezembro de 2018 a fevereiro de 2019. Obteve-se 100 questionários respondidos e validados.

\subsection{Instrumento de Pesquisa}

Esta pesquisa consubstancia-se no instrumento de pesquisa apresentado no Apêndice A. As assertivas foram apresentadas em escala tipo Likert de sete pontos. Utilizou-se o instrumento de pesquisa as questões adaptadas do estudo do Ekholm e Wallin (2011)sobre as utilidades do orçamento. Os autores dividiram as utilidades do orçamento apresentadas por Ax e Kullven (2005) em duas variáveis que foram intituladas planejamento e diálogo. No planejamento, o orçamento deve: estar vinculado às estratégias, coordenar as unidades, alocar recursos e determinar volumes operacionais da organização (Ekholm \& Wallin, 2011). No diálogo, criar consciência do que é importante para se alcançar, comunicar objetivos e ideias, remuneração, atribuição de responsabilidades e motivar o pessoal (Ekholm \& Wallin, 2011).

Para o empoderamento, as três primeiras questões do instrumento de pesquisa foram elaboradas por Spreitzer (1995) e as outras duas foram elaboradas por Speklé, Elten e Widener (2017). As questões averiguaram a independência e a liberdade para fazer escolhas em seu trabalho. O instrumento de pesquisa voltado à criatividade foi pautado por Farmer, Tierney e Kung-McIntyre (2003), com questões que capturam o desenvolvimento de novas ideias e soluções de problemas nos trabalhos dos gestores.

\subsection{Procedimentos de Análise dos Dados}

Os dados foram tratados de modo unidimensional, com consistência interna validada por análise fatorial confirmatória. Para testar as hipóteses se aplicou a técnica de Modelagem de Equações Estruturais, apropriada para compreender relações complexas (Hair Jr Black, Babin, Anderson \& Tatham, 2009). Os parâmetros dessas relações indicam o efeito das variáveis independentes nas variáveis dependentes (Marôco, 2010).

A confiabilidade dos dados também foi calculada pela CompositeReability (CR). O CompositeReability também aceita valores superiores a 0,7 e mede a consistência interna dos itens. Já a validade convergente é calculada pela AverangeVarianceExtracted (AVE), que se refere à quantidade geral de variância nos indicadores e sugere valores acima de 0,5 (Hair Jr et al., 2016).

Além disso, foi realizado o teste de Validade Discriminante (HTMT), o que oferece evidências de que um construto é único e que captura fenômenos que outras medidas desconsideraram. A Validade Discriminante significa, também, que itens individuais medidos devem apresentar apenas um construto latente (Hair et al., 2009). Para a aplicação desse teste, adotou-se o método sugerido por Fornell e Larcker (1981), pois compara a porcentagem da variância extraída para quaisquer dois construtos com a estimativa quadrada da correlação entre esses construtos. As estimativas da variância devem ser maiores que a estimativa quadrática da correlação. Outro indicador que reforça esta validade é o HeterotraitMonotraitRatio (HTMT), sugerido por Henseler et al. (2016). 
Por utilizar dados obtidos a partir de levantamento (survey) com dados das variáveis (exógenas e endógenas) de uma mesma fonte (mesmo respondente, formato de resposta, forma de coleta e no mesmo momento), realizou-se o teste de Harman, conforme pondera Mackenzie e Podsakoff, (2012). Nesse método são desconsiderados o modelo estrutural (relações entre variáveis latentes) e o modelo de mensuração (relações entre os indicadores e as variáveis latentes), o que estima a Análise Fatorial Exploratória (AFE) com a totalidade dos itens em uma mesma análise e que emprega o método de componentes principais não rotacionados (Bido, Mantovani \& Cohen, 2018). Desta forma, o método considera a existência de viés no momento em que a solução resulta em um único fator extraído ou um único fator extrai a maior parte da variância do conjunto de variáveis (Podsakoff, Mackenzie, Lee \& Podsakoff, 2003).

\section{Análise e Discussões dos Resultados}

Primeiramente, apresenta-se a análise descritiva dos dados para permitir compreender o perfil dos respondentes. Assim, tem-se a Tabela 1.

Tabela 1

Estatística descritiva das variáveis da pesquisa

\begin{tabular}{lccccc}
\hline \multicolumn{1}{c}{ Descrição } & Média & Mediana & Mínimo & Máximo & Desvio-padrão \\
\hline Plan_1 & 6,32 & 7,00 & 1,00 & 7,00 & 0,99 \\
\hline Plan_2 & 6,24 & 7,00 & 1,00 & 7,00 & 1,05 \\
\hline Plan_3 & 6,17 & 6,00 & 1,00 & 7,00 & 1,17 \\
\hline Plan_4 & 6,24 & 6,00 & 3,00 & 7,00 & 0,90 \\
\hline Diálog_1 & 6,10 & 7,00 & 1,00 & 7,00 & 1,20 \\
\hline Diálog_2 & 6,11 & 6,00 & 3,00 & 7,00 & 0,93 \\
\hline Diálog_3 & 6,37 & 7,00 & 1,00 & 7,00 & 0,95 \\
\hline Diálog_4 & 5,86 & 6,00 & 1,00 & 7,00 & 1,41 \\
\hline Diálog_5 & 5,70 & 6,00 & 1,00 & 7,00 & 1,57 \\
\hline Emp_1 & 5,82 & 6,00 & 1,00 & 7,00 & 1,33 \\
\hline Emp_2 & 5,57 & 6,00 & 1,00 & 7,00 & 1,64 \\
\hline Emp_3 & 5,78 & 6,00 & 1,00 & 7,00 & 1,40 \\
\hline Emp_4 & 5,58 & 6,00 & 1,00 & 7,00 & 1,63 \\
\hline Emp_5 & 6,21 & 7,00 & 1,00 & 7,00 & 1,17 \\
\hline Criat_1 & 6,24 & 7,00 & 1,00 & 7,00 & 1,07 \\
\hline Criat_2 & 5,94 & 6,00 & 1,00 & 7,00 & 1,23 \\
\hline Criat_3 & 6,15 & 7,00 & 1,00 & 7,00 & 1,31 \\
\hline Criat_4 & 6,39 & 7,00 & 1,00 & 7,00 & 0,96 \\
\hline Criat_5 & 5,94 & 6,00 & 1,00 & 1,27 \\
\hline Nota:Plan & 6700 & \\
\hline
\end{tabular}

Nota: Plan=Planejamento; Dialog=Diálogo; Emp=Empoderamento; Criat=Criatividade.

Fonte: dados da pesquisa.

Em relação à utilidade do planejamento, a assertiva que apresentou maior média $(6,32)$, se refere ao item planejamento. Já na utilidade diálogo, a maior média $(6,37)$ se referiu à assertiva criação de consciência do que é importante para alcançar. No empoderamento, a assertiva que apresentou maior média $(6,21)$ se refere ao alto grau de iniciativa permissível pela organização. No construto da criatividade, a maior média $(6,39)$ tratou da interação entre os gestores concernentes ao desenvolvimento de novas ideias. 
De modo geral, os construtos apresentam médias altas, o que indica que gestores investigados, por um lado percebem o uso do orçamento segregado em diferentes utilidades (planejamento e diálogo) e, por outro, percebem que a organização possibilita um ambiente criativo que desperta o empoderamento.

Foram realizados os testes do mínimo de amostra pelo G-power, no qual os parâmetros foram: tamanho do efeito $(0,15)$; nível de significância de $\alpha=5 \%$; e poder da amostra de 1- $\beta=0,8$ (Faul, Erdfelder, Buchner \& Lang, 2009). O mínimo de respondentes exigido para progredir nas análises é 77 . Verificou-se ainda que as respostas dos $20 \%$ primeiros e os $20 \%$ últimos não apresentaram diferenças significativas, o que indica a não existência do viés da não reposta.

Constatou-se ainda que não existe o viés do método comum, uma vez que após se considerar as variáveis do modelo teórico como dependentes em diferentes momentos, nenhuma delas apresentou itens com VIF superior a 3,3. O mesmo se confirmou pelo teste do fator único de Harman (Gomez-Conde, Lunkes \& Rosa, 2019). Após o atendimento dos critérios, deu-se sequência à modelagem de equações estruturais.

Em seguida, optou-se por elaborar a modelagem de equações estruturais, a qual foi operacionalizada em duas etapas. Na primeira etapa, avalia-se o modelo de mensuração no qual verifica-se a confiabilidade e validade do instrumento de pesquisa (Hair Jr. et al., 2016). O índice de confiabilidade composta atesta a confiabilidade do instrumento quando superiores a 0,70 . Já a AVE, quando superior a 0,50 , confirma a validade convergente, e pelas matrizes Cross Loading e Fornell e Larcker atesta-se a validade descriminante (Hair Jr. et al., 2016). Apresenta-se na Tabela 2 o modelo de mensuração.

Tabela 2

Modelo de mensuração

\begin{tabular}{lcccccc}
\hline \multirow{2}{*}{ Construtos } & \multirow{2}{*}{ CC } & AVE & \multicolumn{4}{c}{ Validade descriminante } \\
\cline { 4 - 7 } & & & Planejamento & Criatividade & Empoderamento & Diálogo \\
\hline Planejamento & 0.755 & 0.518 & 0.720 & & & \\
\hline Criatividade & 0.870 & 0.575 & 0.478 & 0.758 & & \\
\hline Empoderamento & 0.886 & 0.613 & 0.387 & 0.640 & 0.783 & 0.736 \\
\hline Diálogo & 0.822 & 0.541 & 0.394 & 0.536 & 0.437 & \\
\hline
\end{tabular}

Fonte: dados da pesquisa.

Pelos resultados apresentados na Tabela 2, atende-se aos critérios do modelo de mensuração. Confirma-se a confiabilidade dos construtos, uma vez que a confiabilidade composta foi superior a 0,70 (Hair Jr et al. 2016). Atesta-se também a validade dos construtos dado que a AVE foi superior ao limiar de 0,50 e as matrizes de Farnell e Larcker e Cross Loading foram condicentes ao predisposto por Hair Jr et al. (2016). O cumprimento da validade convergente evidencia que são explicados pelos construtos mais de $50 \%$ das variâncias das assertivas que as compõem. E pelo atendimento da validade descriminante observou-se que os construtos são distintos entre si. Verificou-se também ausência de colinearidade entre os itens de cada construto, tendo em vista que o índice VIF ficou inferior a 0,5 em todos os modelos (Hair Jr et al. 2016).

De forma complementar, inicializou-se o Heterotrait-MonotraitRatio (HTMT), conforme sugerido por Henseler et al. (2016), uma vez que reforça a validade dos construtos. Apresenta-se na sequência a rácio HTMT, conforme Tabela 3. 
Tabela 3

Heterotrait-MonotraitRatio (HTMT)

\begin{tabular}{lccc}
\hline \multicolumn{1}{c}{ Construtos } & Planejamento & Criatividade & Empoderamento \\
\hline Criatividade & 0,544 & & \\
\hline Empoderamento & 0,427 & 0,706 & \\
\hline Diálogo & 0,460 & 0,631 & 0,434 \\
\hline
\end{tabular}

Fonte: dados da pesquisa.

Conforme os indicadores na Tabela 3, confirma-se novamente a validade dos construtos, haja vista que foram inferiores ao limiar de 0,85 (Henseler et al. 2016), o que denota diferenciação entre os constructos. Importa salientar que é pré-requisito para a inicialização do bootstraping, o atendimento dos critérios do modelo de mensuração. Confirmado esses requisitos, segue-se com a análise do modelo estrutural.

Em seguida, tem-se o modelo estrutural para servir de parâmetro a rotação de 5.000 subamostras e 5.000 interações, a partir de um intervalo de confiança com enviesamento corrigido e acelerado (biascorrectedandaccelerated) com teste bicaudal ao nível de significância de 5\% (Hair Jr. et al., 2016). Estes parâmetros são inicializados por meio da técnica Bootstraping, que permite confirmar ou não as hipóteses da pesquisa. Evidencia-se na Tabela 4 a relação entre os construtos.

Tabela 4

Relação entre os construtos

\begin{tabular}{|c|c|c|c|c|c|}
\hline Construtos & Coef. & T-Value & P-Value & & Hipótese \\
\hline Planejamento $\rightarrow$ Criatividade & 0.202 & 2.230 & $0.026 * \star$ & $\mathrm{H} 1$ & Confirmada \\
\hline Diálogo $\rightarrow$ Criatividade & 0.261 & 2.731 & $0.006 * * *$ & $\mathrm{H} 2$ & Confirmada \\
\hline Planejamento $\rightarrow$ Empoderamento $\rightarrow$ Criatividade & 0.114 & 1.600 & 0.110 & $\mathrm{H} 3$ & $\begin{array}{c}\text { Não } \\
\text { confirmada }\end{array}$ \\
\hline Diálogo $\rightarrow$ Empoderamento $\rightarrow$ Criatividade & 0.151 & 2.596 & $0.009 * * *$ & $\mathrm{H} 4$ & $\begin{array}{c}\text { Confirmação } \\
\text { parcial }\end{array}$ \\
\hline
\end{tabular}

Nota 1: ${ }^{*}<<0,10 ; * * p<0,05 ; * \star * p<0,01$.

$\mathrm{R}^{2}=$ Criatividade $(0,523) ;$ Empoderamento $(0,246)$

$\mathrm{Q}^{2}=$ Criatividade $(0,240) ;$ Empoderamento $(0,110)$

Fonte: dados da pesquisa.

A hipótese $\mathrm{H} 1$ conjecturou que as utilidades do orçamento agrupadas no planejamento influenciam a criatividade. Os achados demonstram que as utilidades do orçamento agrupadas no planejamento aumentam a criatividade dos gestores de empresas tecnológicas $(\beta=0,202, \mathrm{p}<0,026)$. Ao nível de significância de $5 \%$, essas evidências permitem a não rejeição da H1. Infere-se que a utilidade do orçamento consubstanciada no planejamento induz a uma lógica coercitiva de controle, isso possibilita a padronização dos processos. Essa padronização permite a criação de equilíbrios (Simons, 1995), o que sustenta a criatividade. Ao mesmo tempo em que se dá liberdade aos gestores, também é necessária a padronização das tarefas ao ponto que esses sejam criativos no ambiente de trabalho (Simons, 1995; Adler \& Borys, 1996).

Este achado confirma que o orçamento utilizado para limitar gastos e planejar as metas organizacionais promove o surgimento de alternativas criativas por parte dos gestores para que consigam alcançar resultados satisfatórios, mesmo tendo recursos escassos (Amabile, 1997; Grabner \& Speckbacher, 2016; Cools et al., 2017). Se confirmam as inferências de Wouters e Roijmans (2011) e Speklé et al., (2017), de que o estabelecimento de metas pelo orçamento pode incentivar o surgimento de ideias inovadoras para a solução e a experimentação de problemas. 
Na segunda hipótese, postulou-se que as utilidades do orçamento agrupadas no diálogo exercem maior influência na criatividade do que as utilidades agrupadas no planejamento. Os resultados estatísticos permitem a não rejeição da $\mathrm{H}_{2}$, uma vez que se percebeu que a influência do diálogo na criatividade foi significativa e maior do que a do planejamento $(\beta=0,261, \mathrm{p}<0,006)$. Estes achados permitem inferir que embora a utilidade do orçamento agrupada no planejamento leva à criatividade, a dimensão do diálogo apresenta características habilitantes que oportunizam maior liberdade e independência aos gestores, por isso a influência é maior.

Comprovam-se evidências de que o sentimento autônomo gerado pelo uso habilitante do controle orçamentário é importante para as empresas, contudo, o próprio ambiente de trabalho que promove o diálogo desempenha um papel fundamental no desenvolvimento de habilidades criativas (Amabile et al., 1996; Hirst, Van Knippenberg \& Zhou, 2009; Amabile \& Pillemer, 2012).

Os achados sugerem que o orçamento, como mecanismo de controle habilitante, estimula a interação entre os entes da hierarquia (Ahrens \& Chapman, 2004), o que permite que os gestores se sintam motivados a empreender formas criativas para lidar com as complexas decisões (Wouters \& Wilderom, 2008; Speklé et al., 2017).

A terceira hipótese visou à mediação do empoderamento na relação entre as utilidades do orçamento agrupadas no planejamento e a criatividade. Os achados permitem a rejeição da $\mathrm{H}_{3,}$ dado que o empoderamento não mediou a relação entre a característica coercitiva do orçamento (planejamento) e a criatividade $(\beta=0,114, p<0,110)$. Infere-se que o empoderamento faz com que o uso habilitante do orçamento restrinja o possível efeito direto sobre a criatividade organizacional.

Para tanto, gestores empoderados limitam a liberdade da ação gerencial, tornando o orçamento habilitante, que já se apresenta com falta de diálogo, como um fator que não promove a criatividade (Radke \& Widener, 2016; Ahrens \& Chapman, 2004). Contrariam-se as evidências de que o empoderamento representa a motivação autônoma (Sun, Zhang, Qi \& Chen (2012). Nesse sentido, as organizações que fazem uso do orçamento habilitante precisam ter cuidado com a forma de empoderamento dos gestores, para que não haja um prejuízo no desenvolvimento de atitudes criativas.

Já em relação à $\mathrm{H}_{4}$, que postulou mediação do empoderamento na relação entre as utilidades do orçamento agrupadas no diálogo e a criatividade, confirmou-se a mediação parcial do empoderamento. Conforme apontam Bido e Silva (2019), ocorre a mediação parcial quando o efeito direto apresenta relação significativa ao mesmo tempo em que a relação indireta. Conclui-se que o orçamento, nas utilidades agrupadas no diálogo, comunica as preocupações dos principais gerentes em todas as unidades organizacionais.

Esses funcionários ficam cientes das oportunidades e ameaças potenciais ao atingimento das metas e concebem habilidades criativas para superar os desafios diários (Speklé, Elten \& Widener, 2017). Sendo assim, o controle orçamentário habilitante torna os gestores empoderados, gerando um estímulo aos indivíduos para pensarem em soluções incomuns e abordagens fora do padrão (Burroughs et al., 2011; Cools et al., 2017).

Para tanto, comprovam-se evidências de Hall (2008) e Marginson et al., (2014), em que o uso do orçamento para o diálogo pode ampliar a sensação de empoderamento, isso faz com que gestores experimentem maior liberdade, autonomia e oportunidade para realizar as tarefas. Denota-se que as características habilitantes do orçamento consubstanciadas no diálogo geram maior empoderamento, o que aumenta a criatividade.

As dimensões do orçamento agrupadas no diálogo sugerem que os gestores em ambientes tecnológicos usam o orçamento para maior comunicação dos objetivos, maior conscientização das metas e maior motivação pessoal (Ekholm \& Wallin, 2011). Essas características habilitantes do orçamento aumentam o grau de autonomia (Speklé, Elten \& Widener, 2017) e que geram criatividade (Farmer, Tierney \& Kung-McIntyre, 2003; Speklé, Elten \& Widener,2017). De modo geral, percebe-se queo empoderamento reforça de forma parcial a relação entre o diálogo e a criatividade. 
Quanto ao ajuste do modelo, percebe-se que a criatividade é explicada com grande efeito $\left(R^{2}=0,52\right)$ pelas variáveis independentes do modelo. Em relação à acurácia, a criatividade apresentou um $Q^{2}$ de 0,24 , que aponta pela relevância preditiva do modelo.

\section{Considerações Finais}

$\mathrm{O}$ orçamento permite que haja aumento na criatividade dos gestores, o que independe das suas características de uso. Nesse sentido, sugere-se que a utilização do orçamento, por si só, é um instrumento que oferece criatividade organizacional. Contudo, apesar de ambas as formas de uso do orçamento terem efeito na criatividade, pondera-se que o orçamento de forma habilitante tem maior facilidade de promover a criatividade organizacional, quando comparado com o orçamento coercitivo.

Os resultados também indicam que o empoderamento não tem efeito sobre a relação entre o uso coercitivo do orçamento e a criatividade organizacional. A dificuldade em promover a criatividade pode se relacionar com a falta de diálogo promovido pelo uso coercitivo do orçamento, ocasionado, neste caso, pelo distanciamento criado pelos gestores com sentimento de empoderamento.

Por outro lado, conclui-se que as empresas com uso habilitante do orçamento promovem maior diálogo organizacional, o que permite que as empresas com gestores empoderados tenham efeito inverso dos encontrados nas empresas que só focalizam o orçamento para utilidade de planejamento (coercitivo). Isso confirma o indício de que o empoderamento exerce efeito mediador na relação entre as utilidades do orçamento agrupados no diálogo e a criatividade organizacional.

\subsection{Implicações Teóricas e práticas}

Esta pesquisa gera implicações na literatura ao apresentar que o uso habilitante do orçamento é maior que o uso coercitivo. Percebeu-se que o orçamento estimula maior interação entre os entes hierárquicos (Ahrens \& Chapman, 2004) e também que a presença do empoderamento reforça a relação entre o uso habilitante e a criatividade de maneira parcial.

Isso denota que o orçamento detém características mecanicistas e orgânicas, o que é convergente com a geração de novas ideias, a busca por diferentes formas de resolver problemas, uma maior independência e a busca pela aprendizagem (Ahrens \& Borys, 1996; Ahrens \& Chapman, 2004). Tais ocorrências foram elencadas por Simons (1995) e Ahrens e Borys (1996) ao afirmarem que o controle gerencial aumenta a criatividade.

Do ponto de vista prático, a pesquisa traz implicações ao sugerir que em ambientes dinâmicos, nos quais os indivíduos estão sujeitos à busca por ideias criativas, o uso do orçamento a partir de suas características coercitivas e habilitantes possibilita a concretização dos objetivos organizacionais. Deste modo, permite à organização compreender as formas de uso do orçamento e induzir os gestores à busca por esforços que viabilizem maior empoderamento e criatividade. 


\subsection{Limitações e sugestão para pesquisas futuras}

A pesquisa limita-se às relações propostas, no entanto, análises adicionais poderiam ser inicializadas, a fim de se verificar se as características dos gestores participantes do estudo e as características das organizações em geral exercem algum efeito nas relações propostas. Pelo fato de o orçamento apresentar características que o permitem habilitar ou restringir comportamento, é que se tratou dos termos das características "habilitantes e coercitivas do orçamento". Assim, a proposta expõe-se ao escrutínio de mais pesquisadores na área, ao mesmo tempo em que sugere mais pesquisas que explorem as características habilitantes e coercitivas do orçamento. Estudos futuros podem ainda explorar como o uso o orçamento pode levar a maior aprendizagem organizacional.

\section{Referências}

Abbey, A., \& Dickson, J. W. (1983). R\&D work climate and innovation in semiconductors. Academy of Management Journal, 26(2), pp. 362-368. Doi: https://doi.org/10.2307/255984

Adler, P. S., \& Borys, B. (1996). Two types of bureaucracy: enabling and coercive. Administrative Science Quarterly, 41(1), pp. 61-89. Doi: 10.2307/2393986

Adler, P. S., \& Chen, C. X. (2011). Combining creativity and control: Understanding individual motivation in large-scale collaborative creativity. Accounting, organizations and society, 36(2), pp. 63-85. Doi: https://doi.org/10.1016/j.aos.2011.02.002

Anderson, D. J., Inagaki, H. K., \& Panse, K. M. (2014). Independent, reciprocal neuromodulatory control of sweet and bitter taste sensitivity during starvation in Drosophila. Neuron, 84(4), pp. 806-820. Doi: 10.1016/j.neuron.2014.09.032

Amabile, T. M. (1997). Motivating creativity in organizations: On doing what you love and loving what you do. California management review, 40(1), pp. 39-58. Doi: https://doi.org/10.2307/41165921

Amabile, T. M., Conti, R., Coon, H., Lazenby, J., \& Herron, M. (1996). Assessing the work environment for creativity. Academy of management journal, 39(5), pp. 1154-1184. Doi: https://doi. org/10.5465/256995

Amabile, T. M., \& Pillemer, J. (2012). Perspectives on the social psychology of creativity. The Journal of Creative Behavior, 46(1), pp. 3-15. Doi: 10.1002/jocb.001

Annarelli, A., \& Nonino, F. (2016). Strategic and operational management of organizational resilience: Current state of research and future directions. Omega, 62, pp. 1-18. Doi: 10.1016/j. omega.2015.08.004

Ahrens, T., \& Chapman, C. S. (2004). Accounting for flexibility and efficiency: a field study of management control systems in a restaurant chain*. Contemporary Accounting Research, 21(2), pp. 271-301. Doi: 10.1506/VJR6-RP75-7GUX-XH0X

Alge, B. J., Ballinger, G. A., Tangirala, S., \& Oakley, J. L. (2006). Information privacy in organizations: Empowering creative and extrarole performance. Journal of applied psychology, 91(1), pp. 221. Doi: 10.1037/0021-9010.91.1.221

Appuhami, R. (2017). Exploring the relationship between strategic performance measurement systems and managers' creativity: the mediating role of psychological empowerment and organisational learning. Accounting \& FinanceDoi: 10.1111/acfi.12287 
Arnold, M. C., \& Gillenkirch, R. M. (2015). Using negotiated budgets for planning and performance evaluation: An experimental study. Accounting, organizations and society, 43, pp. 1-16. Doi: https:// doi.org/10.1016/j.aos.2015.02.002

Ax, C., Johansson, C., \& Kullvén, H. (2005). Den nya ekonomistyrningen. 3. painos.

Bedford, R. B. (2015). How low does iron go? Chasing the active species in Fe-catalyzed cross-coupling reactions. Accounts of chemical research, 48(5), pp. 1485-1493. Doi: 10.1021/acs.accounts.5b00042

Beuren, I. M., dos Santos, V., \& Bernd, D. C. (2020). Effects of the Management Control System on Empowerment and Organizational Resilience. Brazilian Business Review.

Bido, D. S., Mantovani, D. M. N., \& Cohen, E. D. (2018). Destruição de escalas de mensuração por meio da análise fatorial exploratória nas pesquisas da área de produção e operações. Gestão é Produção, 25(2), pp. 384-397. Doi: 10.1590/0104-530x3391-16

Bido, D. S., \& da Silva, D. (2019). SmartPLS 3: especificação, estimação, avaliação e relato. Administração: Ensino e Pesquisa, 20(2), pp. 1-31. Doi: 10.13058/raep.2019.v20n2.1545

Burney, L. L., Radtke, R. R., \& Widener, S. K. (2016). The intersection of "bad apples,"'bad barrels," and the enabling use of performance measurement systems. Journal of Information Systems, 31(2), pp. 25-48. Doi: 10.2308/isys-51624

Burroughs, J. E., Dahl, D. W., Moreau, C. P., Chattopadhyay, A., \& Gorn, G. J. (2011). Facilitating and rewarding creativity during new product development. Journal of Marketing, 75(4), pp. 53-67. Doi: $10.2307 / 41228608$

Chapman, C. S., \& Kihn, L. A. (2009). Information system integration, enabling control and performance. Accounting, organizations and society, 34(2), pp. 151-169. Doi: 10.1016/j. aos.2008.07.003

Cools, M., Stouthuysen, K., \& Van den Abbeele, A. (2017). Management control for stimulating different types of creativity: The role of budgets. Journal of Management Accounting Research, 29(3), pp. 1-21. Doi: 10.2308/jmar-51789

Curtis, E., \& Sweeney, B. (2017). Managing different types of innovation: mutually reinforcing management control systems and the generation of dynamic tension. Accounting and Business Research, 47(3), pp. 313-343. Doi: 10.1080/00014788.2016.1255585

Deci, E. L., \& Ryan, R. M. (1987). The support of autonomy and the control of behavior. Journal of personality and social psychology, 53(6), pp. 1024-1037. Doi: 10.1037//0022-3514.53.6.1024

Ekholm, B. G., \& Wallin, J. (2011). The impact of uncertainty and strategy on the perceived usefulness of fixed and flexible budgets. Journal of Business Finance \& Accounting, 38(1-2), pp. 145-164. Doi: 10.1111/j.1468-5957.2010.02228.x

Farmer, S. M., Tierney, P., \& Kung-Mcintyre, K. (2003). Employee creativity in Taiwan: An application of role identity theory. Academy of management Journal, 46(5), pp. 618-630. Doi: https://doi. org/10.2307/30040653

Faul, F., Erdfelder, E., Buchner, A., \& Lang, A. G. (2009). Statistical power analyses using G* Power 3.1: Tests for correlation and regression analyses. Behavior research methods, 41(4), pp. 1149-1160. Doi: 10.3758/BRM.41.4.1149

Fornell, C., \& Larcker, D. F. (1981). Structural equation models with unobservable variables and measurement error: Algebra and statistics. Vol. 18, N. 3, Doi: https://doi.org/10.2307/3150980

Gagné, M., \& Deci, E. L. (2005). Self-determination theory and work motivation. Journal of Organizational behavior, 26(4), pp. 331-362. Doi: 10.1002/job.322 
Grabner, I., \& Speckbacher, G. (2016). The cost of creativity: A control perspective. Accounting, Organizations and Society, 48, pp. 31-42. Doi: 10.1016/j.aos.2015.11.001

Gong, Y., Cheung, S. Y., Wang, M., \& Huang, J. C. (2012). Unfolding the proactive process for creativity: Integration of the employee proactivity, information exchange, and psychological safety perspectives. Journal of management, 38(5), pp. 1611-1633. Doi: https://doi. org/10.1177/0149206310380250

Gomez-Conde, J., Lunkes, R. J., \& Rosa, F. S. (2019). Environmental innovation practices and operational performance. The joint effects of management accounting and control systems and environmental training. Accounting, Auditing \& Accountability Journal. Doi: 10.1108/AAAJ-01-2018-3327

Hall, M. (2008). The effect of comprehensive performance measurement systems on role clarity, psychological empowerment and managerial performance. Accounting, Organizations and Society, 33(2-3), pp. 141-163. Doi: 10.1016/j.aos.2007.02.004

Hair Jr, J. F., Hult, G. T. M., Ringle, C., \& Sarstedt, M. (2016). A primer on partial least squares structural equation modeling (PLS-SEM). Sage publications.

Hair Jr, J. F., Black, W. C., Babin, B. J., Anderson, R. E., \& Tatham, R. L. (2009). Modelagem de Equações Estruturais: uma introdução. Análise multivariada de dados, 6.

Hartmann, F. G., \& Maas, V. S. (2011). The effects of uncertainty on the roles of controllers and budgets: An exploratory study. Accounting and Business Research, 41(5), pp. 439-458. Doi: $10.1080 / 00014788.2011 .597656$

Hempel, P. S., Zhang, Z. X., \& Han, Y. (2012). Team empowerment and the organizational context: Decentralization and the contrasting effects of formalization. Journal of management, 38(2), pp. 475-501. Doi: 10.1177/0149206309342891

Henseler, J., Fassott, G., \& Coelho, P. S. (2016). Testing moderating effects in PLS path models with composite variables. Industrial Management \& Data Systems. pp. 1887-1900. Doi: 10.1108/IMDS06-2016-0248

Henri, J. F. (2006). Management control systems and strategy: A resource-based perspective. Accounting, organizations and society, 31(6), pp. 529-558. Doi: https://doi.org/10.1016/j.aos.2005.07.001

Hirst, G., Van Knippenberg, D., \& Zhou, J. (2009). A cross-level perspective on employee creativity: Goal orientation, team learning behavior, and individual creativity. Academy of management journal, 52(2), pp. 280-293. Doi: 10.5465/AMJ.2009.37308035

Janka, M., \& Guenther, T. W. (2018). Management control of new product development and perceived environmental uncertainty: Exploring heterogeneity using a finite mixture approach. Journal of Management Accounting Research, 30(2), pp. 131-161. Doi: 10.2308/jmar-52019

Kantur, D., \& İşeri-Say, A. (2012). Organizational resilience: A conceptual integrative framework. Journal of Management \& Organization, 18(6), pp. 762-773. Doi: 10.5172/jmo.2012.18.6.762

Koestner, R., Ryan, R. M., Bernieri, F., \& Holt, K. (1984). Setting limits on children's behavior: The differential effects of controlling vs. informational styles on intrinsic motivation and creativity. Journal of personality, 52(3), pp. 233-248. Doi: 10.1111/j.1467-6494.1984.tb00879.x

Kruis, J., \& Maris, G. (2016). Three representations of the Ising model. Scientific reports, 6, 34175.

Kruis, A. M., Speklé, R. F., \& Widener, S. K. (2016). The levers of control framework: An exploratory analysis of balance. Management Accounting Research, 32, pp. 27-44. Doi: https://doi.org/10.1016/j. mar.2015.12.002 
Limnios, E. A. M., Mazzarol, T., Ghadouani, A., \& Schilizzi, S. G. (2014). The resilience architecture framework: four organizational archetypes. European Management Journal, 32(1), pp. 104-116. Doi: https://doi.org/10.1016/j.emj.2012.11.007

MacKenzie, S. B., \& Podsakoff, P. M. (2012). Common method bias in marketing: Causes, mechanisms, and procedural remedies. Journal of retailing, 88(4), pp. 542-555. Doi: 10.1016/j.jretai.2012.08.001

Mahama, H., \& Cheng, M. M. (2013). The effect of managers' enabling perceptions on costing system use, psychological empowerment, and task performance. Behavioral Research in Accounting, 25(1), pp. 89-114. Doi: $10.2308 /$ bria-50333

Marginson, D., McAulay, L., Roush, M., \& van Zijl, T. (2014). Examining a positive psychological role for performance measures. Management Accounting Research, 25(1), pp. 63-75. Doi: https://doi. org/10.1016/j.mar.2013.10.002

Marôco, J. (2010). Análise de Equações Estruturais: Fundamentos teóricos, software \& Aplicações. ReportNumber, Lda.

Müller-Stewens, B., Widener, S. K., Möller, K., \& Steinmann, J. C. (2020). The role of diagnostic and interactive control uses in innovation. Accounting, Organizations and Society, 80, 101078.

Mundy, J. (2010). Creating dynamic tensions through a balanced use of management control systems. Accounting, Organizations and society, 35(5), pp. 499-523. Doi: https://doi.org/10.1016/j. aos.2009.10.005

Podsakoff, P. M., MacKenzie, S. B., Lee, J.-Y., \& Podsakoff, N. P. (2003). Common method biases in behavioral research: A critical review of the literature and recommended remedies. Journal of Applied Psychology, 88(5), pp. 879-903. Doi: 10.1037/0021-9010.88.5.879

Radtke, R. R., \& Widener, S. K. (2016). The Complex World of Control: Integration of Ethics and Uses of Control. In Performance Measurement and Management Control: Contemporary Issues (pp. 1738). Emerald Group Publishing Limited

Ryan, R. M., \& Deci, E. L. (2000). Self-determination theory and the facilitation of intrinsic motivation, social development, and well-being. American psychologist, 55(1), pp. 68-78. Doi: 10.1037/0003066X.55.1.68

Sánchez, J. M., Vélez, M. L., \& Ramón-Jerónimo, M. A. (2012). Do suppliers’ formal controls damage distributors' trust?. Journal of Business Research, 65(7), pp. 896-906. Doi: https://doi.org/10.1016/j. jbusres.2011.06.002

Sierens, E., Vansteenkiste, M., Goossens, L., Soenens, B., \& Dochy, F. (2008). The synergistic relationship of perceived autonomy support and structure in the prediction of self-regulated learning. British Journal of Educational Psychology, 79(1), pp. 57-68. Doi: 10.1348/000709908X304398

Simons, R. (1994). Levers of control: How managers use innovative control systems to drive strategic renewal. Harvard Business Press.

Simons, J. (1995). Foucault and the Political. Psychology Press.

Souza, G. E. de., \& Beuren, I. M. (2018). Reflexos do sistema de mensuração de desempenho habilitante na performance de tarefas e satisfação no trabalho. Revista Contabilidade \& Finanças, 29(77), pp. 194-212. Doi: https://doi.org/10.1590/1808-057x201805850

Souza, G. E. de., Anzilago, M., \& Beuren, I. M. (2017). A Percepção Habilitante dos Sistemas de Custeio pelos Gestores se Reflete no Desempenho de Tarefas?. Revista Contabilidade, Gestão e Governança, 20(3), pp. 416-441. Doi: http://dx.doi.org/10.21714/1984-3925_2017v21n3a6 
Speklé, R. F., van Elten, H. J., \& Widener, S. K. (2013). Creativity and control: A paradox-Evidence from the levers of control framework. Behavioral Research in Accounting, 29(2), pp. 73-96. Doi: 10.2139/ ssrn.2311779

Spreitzer, G. M. (1996). Social structural characteristics of psychological empowerment. Academy of management journal, 39(2), pp. 483-504. Doi: https://doi.org/10.2307/256789

Spreitzer, G. M. (1995). Psychological empowerment in the workplace: Dimensions, measurement, and validation. Academy of management Journal, 38(5), pp. 1442-1465. Doi: https://doi. org/10.5465/256865

Van der Hauwaert, E., \& Bruggeman, W. (2015). The effect of monetary rewards on autonomous motivation in an enabling performance measurement context. Corporate Ownership \& Control, 12(3), pp. 341356. Doi: $10.22495 / \operatorname{cocv} 12 \mathrm{i} 3 \mathrm{c} 3 \mathrm{p} 6$

Wouters, M., \& Roijmans, D. (2011). Using prototypes to induce experimentation and knowledge integration in the development of enabling accounting information. Contemporary Accounting Research, 28(2), pp. 708-736. Doi: https://doi.org/10.1111/j.1911-3846.2010.01055.x

Wouters, M., \& Wilderom, C. (2008). Developing performance-measurement systems as enabling formalization: A longitudinal field study of a logistics department. Accounting, Organizations and Society, 33(4-5), pp. 488-516. Doi: https://doi.org/10.1016/j.aos.2007.05.002

Widener, S. K. (2007). An empirical analysis of the levers of control framework. Accounting, organizations and society, 32(7-8), pp. 757-788. Doi: 10.2139/ssrn.771994 


\section{Apêndice A}

Utilidade do orçamento agrupada no planejamento (Ekholm \& Wallin, 2011)

Para cada uma das assertivas que seguem, indique o quão útil você considera o orçamento para os seguintes propósitos. Considere a escala entre $1=$ Nada útil e 7=Muito Útil

1. Planejamento vinculado às estratégias da empresa

2. Coordenação das unidades da empresa

3. Alocação de recursos para as unidades

4. Determinação de volumes operacionais

Utilidade do orçamento agrupada no diálogo (Ekholm \& Wallin, 2011)

Para cada uma das assertivas que seguem, indique o quão útil você considera o orçamento para os seguintes propósitos. Considere a escala entre $1=$ Nada útil e 7=Muito Útil

1. Atribuição

2. Comunicação de objetivos e ideias

3. Criar consciência do que é importante para alcançar

4. Motivação do pessoal

5. Remuneração

Empoderamento (Spreitzer, 1995; Speklé, Elten e Widener\& 2017)

Para cada uma das assertivas que seguem, indique a independência e liberdade para fazer escolhas em seu trabalho. Considere a escala entre 1 = Não concordo e $7=$ Concordo plenamente

1. Tem autonomia significativa para determinar como fazer o trabalho

2. Decide por si próprio como fazer o trabalho

3. Tem muitas possibilidades de independência e liberdade em como fazer o trabalho

4. É permitido tomar decisões importantes sobre como operar

5. É permitido um alto grau de iniciativa

Criatividade (Farmer, Tierney \& Kung-McIntyre, 2003)

Para cada uma das assertivas que seguem, indique o desenvolvimento de novas ideias e soluções de problemas em seus trabalhos. Considere a escala entre 1= Não concordo e 7= Concordo plenamente

1. Pensa em outras formas de resolver problemas quando encontra obstáculos

2. Tem novas perspectivas sobre problemas antigos

3. Lida com várias novas ideias e problemas ao mesmo tempo

4. Ajuda outras pessoas a desenvolver novas ideias

5. Tem muitas novas ideias 